Results 192 individuals with positive blood culture were included: 89 CROPs and 103 controls. $21 \%$ of cases and $50 \%$ of controls were admitted to the ICU $(\mathrm{p}<0.001)$. The proportion of patients who had positive MEWS of 34 requiring ICU admission was $34.8 \%$ in CROPs compared with $45.6 \%$ in controls $(p=0.129)$. The sensitivity, specificity, positive predictive value, and negative predictive value for a positive MEWS of 34 in CROPs was $52.5 \%, 70 \%, 32.3 \%$, and $84 \%$, respectively, and this was comparable with the control group. ROC analysis showed that MEWS was a significant predictor for ICU admission if calculated 12 to 36 hours before positive blood culture in CROPs, and a threshold of 33 had the best specificity (86-91\%) for predicting ICU admission, whereas a threshold of 34 was more suitable for controls. MEWS was generally a poor predictor for mortality.

Conclusion MEWS in general has weak discriminatory value in predicting ICU admission in CROPs. A threshold of 33 MEWS at 12 to 36 hours before positive blood culture was found to be the best cutoff for predicting ICU admission in CROPs compared with a threshold of 34 in controls. MEWS was a poor predictor for mortality within 28 days. The combination of MEWS with clinical judgment might improve prediction for ICU admission.

\section{ACCELERATING HEMOGLOBIN (HBA1C) TEST RESULTS IN FOLLOW-UP DIABETIC CLINICS AT A PRIMARY HEALTHCARE (PHC) CENTER USING THE POINT-OF- CARE HBA1C TESTING DEVICE}

Faris Nabil Fatani, Kkaled Alsurimi. College of Public Health and Health Informatics, King Saud bin Abdulaziz University for Health Sciences

\subsection{6/bmjoq-2019-PSF.4}

Background Based on daily manual patient registry data, as of September 2018, we found that all follow-up patients with diabetes (100\%) at Almasif Primary Care Center in Northern Riyadh had to wait an average of 3.5 days for their hemoglobin A1C (HbA1c) results in order for their physician to make any decisions on their treatment plan. One of the main quality dimensions is the improvement of the timeliness of healthcare, including reducing unnecessary waiting that might cause a harmful delay for patient and providers. Thus, creating a lean management process for diabetic patient work-up will lead to better care in terms of awareness, diagnosis, and treatment, and improve the quality of diabetic patient care in primary healthcare. The setting of this project was Almasif Primary Health Care Center, located in Northern Riyadh, Saudi Arabia. The improvement aim was to accelerate the diabetic care follow-up process by removing the non-added value and decreasing the long waiting time for $\mathrm{HbA1c}$ results to less than 15 minutes on the same day of the visit among at least $80 \%$ of patients with diabetes attending Almasif Primary Health Care Center, Northern Riyadh, by November 2018.

Methods A multidisciplinary quality team has been formed. The team used several quality tools, such as brainstorming technique, process mapping, and cases-affect diagnosis, among others. Improvement measures included the percentage of patients with diabetes who had their treatment care plan changed due to HbA1c as the outcome measure, and the percentage of patients who had the HbA1c result in 10 minutes as the process measure. Several rapid PDSA (plando-study-act) cycles have been conducted to test the change idea of the point-of-care HbA1c testing device. The idea worked well and data have been analyzed and presented on a run chat showing the changes made (PDSA cycles) and improvement over time using the process and outcome measures.

Results All follow-up diabetic patients (17 [100\%] of 17) got their HbA1c results within less than 15 minutes. And more importantly, more than half (nine [53\%] of 17) of the diabetic patients had their treatment plan changed on the same day of the visit. The majority of the diabetic clinic's nurses were satisfied (eight out of ten) and competent to perform the new task.

Conclusion The idea of using the point-of-care HbA1c testing device is very promising to improve the quality and safety of follow-up of diabetic patients at the primary healthcare clinic. It is highly recommended to replicate the idea nationally.

\section{ACQUISITION OF CARBAPENEM-RESISTANT KLEBSIELLA PNEUMONIAE DETECTED BY ACTIVE SURVEILLANCE TESTING IN ADULT INTENSIVE CARE UNIT IN RIYADH, SAUDI ARABIA}

Ayham Salah Albadawi, Yahya AlBakheet, Kassem Abou Yassine, Eman AlGhamdi, Angela Caswell, Saja Marhoun, Aiman El-Saed, Majid Alshamrani, Hanan Balkhy. Infection Prevention and Control King Abdulaziz Medical City - Riyadh

\subsection{6/bmjoq-2019-PSF.5}

Background During the last quarter of 2017, there was an increase in the number of patients with carbapenem-resistant Klebsiella pneumoniae (CRKP) in the adult intensive care unit (AICU) at King Abdullah Specialist Children Hospital, Riyadh, Saudi Arabia. Worldwide, invasive infections caused by carbapenem-resistant Enterobacteriaceae, including CRKP, have been associated with high morbidity and mortality. The target population at AICU is mainly oncology patients who need critical care. It has been suggested that active surveillance testing (AST) can help to minimize exposure within selected units. Additionally, it can estimate the percentage of within-unit acquisition of CRKP. The objective of the current study was to estimate the acquisition of CRKP and the compliance with AST.

Methods AST was done to all patients admitted to the AICU between January 2018 to December 2018 and to those who were discharged, provided that no positive AST or clinical results were documented at admission or during the unit stay. Acquisition of CRKP was defined as positive CRKP (detected by AST or clinically) after an initial negative finding during the first 3 days of unit stay. Compliance of admission AST was defined as testing rectal specimens obtained during the first 3 days of unit stay among all admitted patients. Compliance of discharge AST was defined as testing rectal specimens obtained at discharge or after the first 3 days of unit stay among non-prevalent patients.

Results During the study period, 375 (90.1\%) of 416 admitted patients had AST at admission. Of the 375, 180 (48.0\%) were eligible for discharge AST. $87(48.3 \%)$ of the 180 eligible patients had AST at discharge. The prevalence of positive CRKP at admission was $1.9 \%$ (seven of 375). Acquisition of CRKP during the unit stay was 3.4\% (three of 87). Of 416 
patients admitted to the AICU, 30.5\% died, $69.0 \%$ were transferred to another unit, and $0.5 \%$ were discharged.

Conclusion The findings showed high compliance of AST at admission but low compliance at discharge, which needs further enforcement. Although the acquisition of CRKP was only $3.4 \%$, it represents adding almost double (1.8) new cases of CRKP to the admission level. Future research is required to assess the impact of AST on the burden of healthcare-associated infections.

\section{EVALUATION OF ORAL ANTICANCER MEDICATION HANDLING, STORAGE, AND DISPOSAL PRACTICES AMONG CANCER PATIENTS AND THEIR CAREGIVERS IN THE HOME SETTING AT PRINCESS NORAH ONCOLOGY CENTER}

Ashwag A Algethami, Majed A Alshamrani, Atika M AlHarbi, Aeshah A AlAzmi, Mansoor A Khan. Pharmaceutical Care Services Department, Ministry of National Guard Health Affairs, King Abdulaziz Medical City Jeddah, Saudi Arabia

\subsection{6/bmjoq-2019-PSF.6}

Background Oral chemotherapeutic agents are hazardous drugs that are commonly prescribed for a variety of indications. Using oral anticancer medications may ensure better therapy adherence, but there is not much knowledge about the safety and risks of accidental exposure with subsequent fatalities. Unintentional exposure can take place at different phases of treatment by splitting, crushing, transporting, unpacking, storage, and disposal. Although there are several well-established guidelines for safe handling of parenteral chemotherapy in the healthcare setting, there are few recommendations concerning proper handling of oral chemotherapy at home for patient and environmental safety. This survey aimed to evaluate the handling, storage, and disposal practices of oral anticancer medications among cancer patients and their caregivers in the home setting.

Methods A questionnaire-based cross-sectional study was done in all adult cancer patients/patient caregivers receiving oral anticancer medications and visiting the oncology outpatient pharmacy. Oncology pharmacists interviewed patients after obtaining consent. Survey responses were analysed using descriptive statistics.

Results A total of 201 participants agreed to be interviewed, of whom most were female (67\%). Nearly $44 \%$ of participants were aged between 40 and 60 years. The majority of participants were educated (157 responses, 78\%) while only $44(22 \%)$ had never been to school. The top five oral anticancer medications taken by patients were tamoxifen, capecitabine, letrozole, dasatinib, and imatinib. All participants reported that medications were kept away from children and pets (100\%). $196(97.5 \%)$ patients responded that the medication was stored away from extreme heat, cold, and humidity. $195(97 \%)$ patients reported keeping their medications in the original container. Hand washing and wearing gloves were not a consistent practice among patients and caregivers. Among all participants, only nine (4.5\%) reported 'always' wearing gloves; 48 (24\%) reported 'always' washing hands after handling anticancer medications. Patients and caregivers reported that they had been informed about safe handling and storage by their physician (47\%) and pharmacist (30\%), while $40 \%$ of them had not been informed. In terms of disposal practice, more than half $(66 \%)$ of patients had not had unused or expired medications, $29 \%$ of patients dispose of them in the trash, and $27 \%$ return them to the pharmacy or doctor's office.

Conclusion Our findings, while preliminary, suggest that patients' and caregivers' awareness regarding handling practices are inconsistent with the published recommendations. Appropriate and comprehensive education is needed to mitigate exposure risk to these agents in the home setting.

\section{REDUCE MEDICATION ADMINISTRATION DELAY IN NEONATAL INTENSIVE CARE UNIT}

Noura Alshami, Amerah NAl Saleh, Amjed Abu Alburak, Regina Manlulu, Saif Al Saif, Mark Anthony Castro, Eden Grace Abainza, Suzan Ziyad Omran, Yvonne Marlene Makins, Mary Ann Rivera, Flordelisa Ramos. Neonatal Intensive Care Unit, King Abdulaziz Medical City Riyadh, Ministry of National Guard Health Affairs

\subsection{6/bmjoq-2019-PSF.7}

Background Medication errors are the most common medical errors experienced by patients. Neonates are even more vulnerable to medication errors because of drug dosing that is influenced by weight, gestational age, and postnatal age. Data obtained from the safety reporting system related to medication and fluids events in the neonatal intensive care unit (NICU) from January to August 2017 indicated a high rate of near-misses and errors reaching patients. The top five specific event types related to medication and fluids events were, in the following order: delay, incorrect dose, incorrect frequency, medication packaging issues, and dose omitted.

Methods In September 2017, a project team was formed including members from the following disciplines: neonatology, neonatal nursing, pharmacy, medication safety program, and quality department. Data obtained from the safety reporting system were examined and analyzed. After thorough analysis of the data, the project team agreed to tackle the delay in STAT medication administration in the first cycle of the project. The team also completed a process map and cause and effect diagram to understand the root causes of the delay. The average time required for a STAT medication or fluid to be administered to the NICU patient was 3 hours, and the delay in STAT medication was mainly related to septic shock and suspected sepsis management. To reduce the delay in STAT medication administration, the NICU must be provided with a floor stock of pre-mixed antibiotics that is readily available for administration when required. A standardized order set for septic shock doses was programed in the BestCare System to reduce the time and effort required in generating orders. All the stakeholders involved in the process were made aware of the new changes and a departmental policy was created to guide the staff practice.

Results After the implementation of the pre-mixed antibiotic floor stock and pre-set orders, the turnaround time for administration of STAT medication in the NICU reduced from 3 hours to 1 hour, and there was a $60 \%$ reduction in the delay in medication administration events.

Conclusion The new changes in the process impacted positively on patient safety and prevention of harm. They reduced time and redundancy in the previous process. The changes also improved staff satisfaction and reduced the tension that used to result from the frequent communication and followups involved until the appropriate medication was made available for administration. 